Korean J. Math. 20 (2012), No. 3, pp. 307-314

\title{
PROPERTIES OF INDUCED INVERSE POLYNOMIAL MODULES OVER A SUBMONOID
}

\author{
Eunha Cho And Jinsun JeOnG*
}

\begin{abstract}
Let $M$ be a left $R$-module and $R$ be a ring with unity, and $S=\{0,2,3,4, \cdots\}$ be a submonoid. Then $M\left[x^{-s}\right]=\left\{a_{0}+\right.$ $\left.a_{2} x^{-2}+a_{3} x^{-3}+\cdots+a_{n} x^{-n} \mid a_{i} \in M\right\}$ is an $R\left[x^{s}\right]$-module. In this paper we show some properties of $M\left[x^{-s}\right]$ as an $R\left[x^{s}\right]$-module.

Let $f: M \longrightarrow N$ be an $R$-linear map and $\bar{M}\left[x^{-s}\right]=\left\{a_{2} x^{-2}+\right.$ $\left.a_{3} x^{-3}+\cdots+a_{n} x^{-n} \mid a_{i} \in M\right\}$ and define $N+\bar{M}\left[x^{-s}\right]=\left\{b_{0}+\right.$ $\left.a_{2} x^{-2}+a_{3} x^{-3}+\cdots+a_{n} x^{-n} \mid b_{0} \in N, a_{i} \in M\right\}$. Then $N+\bar{M}\left[x^{-s}\right]$ is an $R\left[x^{s}\right]$-module.

We show that given a short exact sequence $0 \longrightarrow L \longrightarrow M \longrightarrow$ $N \longrightarrow 0$ of $R$-modules, $0 \longrightarrow L \longrightarrow M\left[x^{-s}\right] \longrightarrow N+\bar{M}\left[x^{-s}\right] \longrightarrow 0$ is a short exact sequence of $R\left[x^{s}\right]$-module. Then we show $E_{1}+\overline{E_{0}}\left[x^{-s}\right]$ is not an injective left $R\left[x^{s}\right]$-module, in general.
\end{abstract}

\section{Introduction}

Let $M$ be a left $R$-module and $R$ be a ring with unity and $S=$ $\{0,2,3,4, \cdots\}$ be a submonoid. Then $M\left[x^{s}\right]=\left\{a_{0}+a_{2} x^{2}+a_{3} x^{3}+\cdots+\right.$ $\left.a_{n} x^{n} \mid a_{i} \in M\right\}$ is an $R\left[x^{s}\right]$-module defined by

$x^{k}\left(a_{0}+a_{2} x^{2}+a_{3} x^{3}+\cdots+a_{n} x^{n}\right)=a_{0} x^{k}+a_{2} x^{2+k}+a_{3} x^{3+k}+\cdots+a_{n} x^{n+k}$

for $x^{k} \in R\left[x^{s}\right]$

Also $M\left[x^{-s}\right]=\left\{a_{0}+a_{2} x^{-2}+a_{3} x^{-3}+\cdots+a_{n} x^{-n} \mid a_{i} \in M\right\}$ is an $R\left[x^{s}\right]$-module defined by

$x^{k}\left(a_{0}+a_{2} x^{-2}+a_{3} x^{-3}+\cdots+a_{n} x^{-n}\right)=a_{k}+a_{k+2} x^{-2}+a_{k+3} x^{-3}+\cdots+a_{n} x^{-n+k}$

Received July 2, 2012. Revised August 30, 2012. Accepted September 5, 2012.

2010 Mathematics Subject Classification: 16E30, 13C11, 16D80.

Key words and phrases: injective module, inverse polynomial modules, induced module.

${ }^{*}$ Corresponding author. 
for $x^{k} \in R\left[x^{s}\right]$. For example,

$x^{3}\left(a_{0}+a_{2} x^{-2}+a_{3} x^{-3}+\cdots+a_{n} x^{-n}=a_{3}+a_{5} x^{-2}+a_{6} x^{-3}+\cdots+a_{n} x^{-n+3}\right.$.

Induced inverse polynomial module was introduced in ([8]). Let $M$ and $N$ be left $R$-modules and $f: M \longrightarrow N$ be an $R$-linear map. Then an induced polynomial $N+x^{-1} M\left[x^{-1}\right]$ is a left $R[x]$-module defined by

$$
x\left(b_{0}+a_{1} x^{-1}+\cdots+a_{n} x^{-n}\right)=b_{1}+a_{2} x^{-1}+\cdots+a_{n} x^{-n+1},
$$

where $f\left(a_{1}\right)=b_{1}, b_{0} \in N$, and $a_{i} \in M$. In this paper we generalized induced polynomial modules over a submonoid.

Let $f: M \longrightarrow N$ be an $R$-linear map and let $\bar{M}\left[x^{-s}\right]=\left\{a_{2} x^{-2}+\right.$ $\left.a_{3} x^{-3}+\cdots+a_{n} x^{-n} \mid a_{i} \in M\right\}$ and define $N+\bar{M}\left[x^{-s}\right]=\left\{b_{0}+a_{2} x^{-2}+\right.$ $\left.a_{3} x^{-3}+\cdots+a_{n} x^{-n} \mid b_{0} \in N, a_{i} \in M\right\}$. Then $N+\bar{M}\left[x^{-s}\right]$ is an $R\left[x^{s}\right]$ module defined by

$$
\begin{aligned}
& x^{k}\left(b_{0}+a_{2} x^{-2}+a_{3} x^{-3}+\cdots+a_{n} x^{-n}\right) \\
& \quad=f\left(a_{k}\right)+a_{k+2} x^{-2}+a_{k+3} x^{-3}+\cdots+a_{n} x^{-n+k} .
\end{aligned}
$$

For example,

$x^{2}\left(b_{0}+a_{2} x^{-2}+a_{3} x^{-3}+\cdots+a_{n} x^{-n}\right)=f\left(a_{2}\right)+a_{4} x^{-2}+a_{5} x^{-3}+\cdots+a_{n} x^{-n+2}$.

A left $R$-module $E$ is said to be injective if given any injective linear map $\sigma: M^{\prime} \longrightarrow M$ and any linear map $h: M^{\prime} \longrightarrow E$, there is a linear map $g: M \longrightarrow E$ such that $g \circ \sigma=h$. That is,

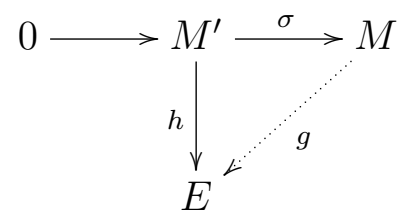

can always be completed to a commutative diagram $([9])$.

The map $f: x^{2} E\left[x^{s}\right] \longrightarrow E\left[x^{s}\right]$ defined by $f\left(e x^{2}\right)=e$ is an $R\left[x^{s}\right]$ linear map. So consider the following diagram

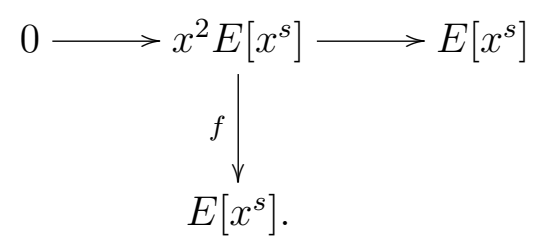


Then we easily see that the above diagram can not be completed for any $R\left[x^{s}\right]$-linear map. Thus $E\left[x^{s}\right]$ is not an injective left $R\left[x^{s}\right]$-module with $E \neq 0$.

Northcott([3]) defined inverse polynomial modules and used inverse polynomial modules to study the properties of injective modules and he studied $K\left[x^{-1}\right]$ as $K[x]$-module on field $K$. And McKerraw ([2]) showed that if $R$ is a left noetherian ring and $E$ is an injective left $R$-module, then $E\left[x^{-1}\right]$ is an injective envelope of $M\left[x^{-1}\right]$ as $R[x]$-module. Inverse polynomial modules were studied in $([4]),([5])$ and recently in $([1]),([5])$, $([6]),([7])$.

\section{2. inverse polynomial modules over a submonoid}

Through this paper we let $S=\{0,2,3,4, \cdots\}$ a submonoid.

DeFinition 2.1. Let $M$ be a left $R$-module and $R$ be a ring with unity and $S=\{0,2,3,4, \cdots\}$ be a submonoid. Then $M\left[x^{-s}\right]=\left\{a_{0}+\right.$ $\left.a_{2} x^{-2}+a_{3} x^{-3}+\cdots+a_{n} x^{-n} \mid a_{i} \in M\right\}$ is an $R\left[x^{s}\right]$-module defined by

$r\left(a_{0}+a_{2} x^{-2}+a_{3} x^{-3}+\cdots+a_{n} x^{-n}\right)=r a_{0}+r a_{2} x^{-2}+r a_{3} x^{-3}+\cdots+r a_{n} x^{-n}$ and

$x^{k}\left(a_{0}+a_{2} x^{-2}+a_{3} x^{-3}+\cdots+a_{n} x^{-n}\right)=a_{k}+a_{k+2} x^{-2}+a_{k+3} x^{-3}+\cdots+a_{n} x^{-n+k}$ where $r \in R$ and $x^{k} \in R\left[x^{s}\right]$.

Proposition 2.2. Let $\phi: M\left[x^{-s}\right] \longrightarrow M\left[x^{-s}\right]$ be an $R\left[x^{s}\right]$-linear map. Then $\phi(M) \subset\left(M+M x^{-2}\right) \cap\left(M+M x^{-3}\right)=M$.

Proof. Suppose $m \in M$ and $\phi(m)=a_{0}+a_{2} x^{-2}+a_{3} x^{-3}+\cdots+a_{n} x^{-n}$. Then for $x^{2} \in R\left[x^{s}\right], \phi\left(x^{2} m\right)=\phi(0)=0$, and $x^{2} \phi(m)=a_{2}+a_{4} x^{-2}+$ $\cdots+a_{n} x^{-n+2}=0$ implies $a_{2}=a_{4}=\cdots=a_{n}=0$. Therefore, $\phi(M) \subset$ $\left(M+M x^{-2}\right) \cap\left(M+M x^{-3}\right)=M$.

If $N$ is a submodules of $M$, then we easily see

$$
\frac{M\left[x^{-s}\right]}{N\left[x^{-s}\right]} \cong \frac{M}{N}\left[x^{-s}\right]
$$

as $R\left[x^{s}\right]$-module. 
Proposition 2.3. Let $M$ be a left $R$-module, then

$$
\frac{M\left[x^{-s}\right]}{M+M x^{-3}} \cong M\left[x^{-s}\right] \text {. }
$$

Proof. Define $\phi: M\left[x^{-s}\right] \longrightarrow M\left[x^{-s}\right]$ by

$$
\begin{aligned}
& \phi\left(a_{0}+a_{2} x^{-2}+a_{3} x^{-3}+\cdots+a_{n} x^{-n}\right) \\
& =\phi\left(a_{0}+a_{2} x^{-2}+a_{3} x^{-3}+\cdots+a_{n} x^{-n}\right) \\
& =a_{2}+a_{4} x^{-2}+\cdots+a_{n} x^{-n+2} .
\end{aligned}
$$

Then we easily see that $\phi$ is an $R\left[x^{s}\right]$-linear map.

Let $a_{0}+a_{2} x^{-2}+a_{3} x^{-3}+\cdots+a_{n} x^{-n} \in M\left[x^{-s}\right]$, then $\phi\left(a_{0} x^{-2}+a_{2} x^{-4}+a_{3} x^{-5}+\cdots+a_{n} x^{-n-2}\right)=a_{0}+a_{2} x^{-2}+a_{3} x^{-3}+\cdots+a_{n} x^{-n}$. Thus $\phi$ is surjective.

Let $a_{0}+a_{3} x^{-3} \in M\left[x^{-s}\right]$, then $\phi\left(a_{0}+a_{3} x^{-3}\right)=0$, so that $M+M x^{-3} \subset$ $\operatorname{ker}(\phi)$. Let $a_{0}+a_{2} x^{-2}+a_{3} x^{-3}+\cdots+a_{n} x^{-n} \in \operatorname{ker}(\phi)$, then

$\phi\left(a_{0} x^{-2}+a_{2} x^{-4}+a_{3} x^{-5}+\cdots+a_{n} x^{-n-2}\right)=a_{2}+a_{4} x^{-2}+\cdots+a_{n} x^{-n+2}=0$, so that $a_{2}=a_{4}=a_{5}=\cdots=a_{n}=0$, so that $\operatorname{ker}(\phi) \subset M+M x^{-3}$. Thus $\operatorname{ker}(\phi)=M+M x^{-3}$. Therefore,

$$
\frac{M\left[x^{-s}\right]}{M+M x^{-3}} \cong M\left[x^{-s}\right] .
$$

Proposition 2.4. Let $M$ be a left $R$-module, then

$$
\sigma: \frac{M\left[\left[x^{-s}\right]\right]}{M\left[x^{-s}\right]} \longrightarrow \frac{M\left[\left[x^{-s}\right]\right]}{M\left[x^{-s}\right]}
$$

by $\sigma\left(f+M\left[x^{-s}\right]\right)=x^{2}\left(f+M\left[x^{-s}\right]\right)$ is an isomorphism. Then

Proof. Let $f+M\left[x^{-s}\right] \in \operatorname{ker}(\sigma)$ and let $f=a_{0}+a_{2} x^{-2}+a_{3} x^{-3}+\cdots$.

$$
\begin{aligned}
\sigma\left(f+M\left[x^{-s}\right]\right) & =x^{2}\left(f+M\left[x^{-s}\right]\right) \\
& =a_{2}+a_{4} x^{-2}+a_{5} x^{-3}+\cdots+M\left[x^{-s}\right] \\
& =M\left[x^{-s}\right] .
\end{aligned}
$$

So $a_{k} x^{-k+2}+a_{k+1} x^{-k+1}+\cdots=0, a_{k}=a_{k+1}=\cdots=0$, for some $k$. Thus $f+M\left[x^{-s}\right]=M\left[x^{-s}\right]$. Therefore, $\sigma$ is injective. 
Let $f+M\left[x^{-1}\right]=\left(a_{0}+a_{2} x^{-2}+a_{3} x^{-3}+\cdots\right)+M\left[x^{-s}\right] \in \frac{M\left[\left[x^{-s}\right]\right]}{M\left[x^{-s}\right]}$. Then there exists

$g+M\left[x^{-s}\right]=\left(a_{0} x^{-2}+a_{2} x^{-4}+a_{3} x^{-5}+\cdots+a_{k} x^{-k-2}+\cdots\right)+M\left[x^{-s}\right]$ such that $\sigma\left(g+M\left[x^{-s}\right]\right)=f+M\left[x^{-s}\right]$. Therefore, $\sigma$ is surjective. Hence, $\sigma$ is an isomorphism.

\section{Induced polynomial modules over a submonoid}

Definition 3.1. Let $f: M \longrightarrow N$ be an $R$-linear map and $S=$ $\{0,2,3,4, \cdots\}$ be a submonoid. Then $N+\bar{M}\left[x^{-s}\right]$ is an $R\left[x^{s}\right]$-module, where $\bar{M}\left[x^{-s}\right]=\left\{a_{2} x^{-2}+a_{3} x^{-3}+\cdots+a_{n} x^{-n} \mid a_{i} \in M\right\}$ be defined by $r\left(b_{0}+a_{2} x^{-2}+a_{3} x^{-3}+\cdots+a_{n} x^{-n}\right)=r b_{0}+r a_{2} x^{-2}+r a_{3} x^{-3}+\cdots+r a_{n} x^{-n}$ and $x^{k}\left(b_{0}+a_{2} x^{-2}+a_{3} x^{-3}+\cdots+a_{n} x^{-n}\right)=f\left(a_{k}\right)+a_{k+2} x^{-2}+a_{k+3} x^{-3}+\cdots+a_{n} x^{-n+k}$ for $x^{k} \in R\left[x^{s}\right], b_{0} \in N$. Similarly, we can define

$$
N+\bar{M}\left[x^{-s}\right]=\left\{b_{0}+a_{2} x^{-2}+a_{3} x^{-3}+\cdots+a_{n} x^{-n} \mid b_{0} \in N, a_{i} \in M\right\}
$$

as a left $R\left[x^{s}\right]$-module.

Theorem 3.2. If $0 \rightarrow L \rightarrow M \rightarrow N \rightarrow 0$ is a short exact sequence of $R$-modules, then

$$
0 \rightarrow L \rightarrow M\left[x^{-s}\right] \rightarrow N+\bar{M}\left[x^{-s}\right] \rightarrow 0
$$

is a short exact sequence of $R\left[x^{s}\right]$-module.

Proof. Let $f\left[x^{-s}\right]: L \longrightarrow M$ be defined by $f\left[x^{-s}\right](l)=f(l)$ for $l \in$ $L$. Then easily $f\left[x^{-s}\right]$ is an injective $R\left[x^{s}\right]$-linear map. Let $g\left[x^{-s}\right]$ : $M\left[x^{-s}\right] \rightarrow N+\bar{M}\left[x^{-s}\right]$ be defined by

$g\left[x^{-s}\right]\left(a_{0}+a_{2} x^{-2}+a_{3} x^{-3}+\cdots+a_{n} x^{-n}\right)=g\left(a_{0}\right)+a_{2} x^{-2}+a_{3} x^{-3}+\cdots+a_{n} x^{-n}$ Then easily $g\left[x^{-s}\right]$ is an $R\left[x^{s}\right]$-linear map.

Let $b_{0}+a_{2} x^{-2}+a_{3} x^{-3}+\cdots+a_{n} x^{-n} \in N+\bar{M}\left[x^{-s}\right]$. Then since $g$ is a surjective $R$-linear map, there exists $a_{0} \in M$ such that $g\left(a_{0}\right)=b_{0}$. Thus $g\left[x^{-s}\right]\left(a_{0}+a_{2} x^{-2}+a_{3} x^{-3}+\cdots+a_{n} x^{-n}\right)=b_{0}+a_{2} x^{-2}+a_{3} x^{-3}+\cdots+a_{n} x^{-n}$. So $g\left[x^{-s}\right]$ is a surjective $R\left[x^{s}\right]$-linear map.

Now $\left(g\left[x^{-s}\right] \circ f\left[x^{-s}\right]\right)(l)=g\left[x^{-s}\right](f(l))=g(f(l))=0$, so that

$$
\operatorname{Im}\left(f\left[x^{-s}\right]\right) \subseteq \operatorname{Ker}\left(g\left[x^{-s}\right]\right) .
$$


And if $\left.a_{0}+a_{2} x^{-2}+a_{3} x^{-3}+\cdots+a_{n} x^{-n}\right) \in \operatorname{Ker}\left(g\left[x^{-s}\right]\right)$, then

$$
\begin{aligned}
& g\left[x^{-s}\right]\left(a_{0}+a_{2} x^{-2}+a_{3} x^{-3}+\cdots+a_{n} x^{-n}\right) \\
& =g\left(a_{0}\right)+a_{2} x^{-2}+a_{3} x^{-3}+\cdots+a_{n} x^{-n} \\
& =0
\end{aligned}
$$

implies $g\left(a_{0}\right)=0$, and $a_{2}=a_{3}=\cdots=a_{n}=0$. Thus $a_{0} \in \operatorname{Ker}(g)=$ $\operatorname{Im}(f)$, so that $\operatorname{Ker}\left(g\left[x^{-s}\right]\right) \subseteq \operatorname{Im}\left(f\left[x^{-s}\right]\right)$. Therefore, $\operatorname{Im}\left(f\left[x^{-s}\right]\right) \subseteq$ $\operatorname{Ker}\left(g\left[x^{-s}\right]\right)$. Hence,

$$
0 \rightarrow L \rightarrow M\left[x^{-s}\right] \rightarrow N+\bar{M}\left[x^{-s}\right] \rightarrow 0
$$

is a short exact sequence of $R\left[x^{s}\right]$-modules.

Theorem 3.3. Let $0 \longrightarrow N \stackrel{f}{\longrightarrow} E_{0} \stackrel{g}{\longrightarrow} E_{1} \longrightarrow 0$ be a short exact sequence of $R$-modules, with $\operatorname{inj}_{\operatorname{dim}_{R}} N=1$, where $E_{0}, E_{1}$ are injective left $R$-modules. Then $E_{1}+\overline{E_{0}}\left[x^{-s}\right]$ is not an injective left $R\left[x^{s}\right]$-module.

Proof. Suppose $E_{1}+\overline{E_{0}}\left[x^{-s}\right]$ is an injective left $R\left[x^{s}\right]$-module. Then we can complete the following diagram

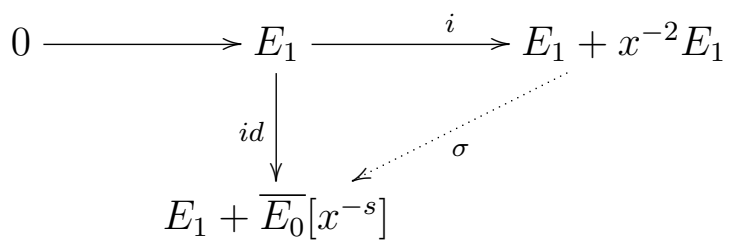

as commutative diagram by an $R\left[x^{s}\right]$-linear map $\sigma$. Then there exists an $R$-linear map $h: E_{1} \longrightarrow E_{0}$ such that $g \circ h=i d_{E_{1}}$. This contradicts the fact that the short exact sequence $0 \longrightarrow N \stackrel{f}{\longrightarrow} E_{0} \stackrel{g}{\longrightarrow} E_{1} \longrightarrow 0$ is not split. Hence, $E_{1}+\overline{E_{0}}\left[x^{-s}\right]$ is not an injective left $R\left[x^{s}\right]$-module.

Definition 3.4. Let $M$ be a left $R$-module and $S=\{0,2,3,4, \cdots\}$ be a submonoid, then $M\left[x^{s}, x^{-s}\right]=\left\{a_{0}+a_{2} x^{2}+a_{3} x^{3}+\cdots+a_{i} x^{i}+b_{2} x^{-2}+\right.$ $\left.b_{3} x^{-3}+\cdots+b_{j} x^{-j} \mid a_{n}, b_{m} \in M\right\}$ is a left $R\left[x^{s}\right]$-module be defined by

$$
\begin{aligned}
& r\left(a_{0}+a_{2} x^{2}+a_{3} x^{3}+\cdots+a_{i} x^{i}+b_{2} x^{-2}+b_{3} x^{-3}+\cdots+b_{j} x^{-j}\right) \\
& \quad=r a_{0}+r a_{2} x^{2}+r a_{3} x^{3}+\cdots+r a_{i} x^{i}+r b_{2} x^{-2}+r b_{3} x^{-3}+\cdots+r b_{j} x^{-j}
\end{aligned}
$$


and

$$
\begin{aligned}
& x^{k}\left(a_{0}+a_{2} x^{2}+a_{3} x^{3}+\cdots+a_{i} x^{i}+b_{2} x^{-2}+b_{3} x^{-3}+\cdots+b_{j} x^{-j}\right) \\
& =a_{0} x^{k}+a_{2} x^{2+k}+\cdots+r a_{i} x^{i+k}+b_{2} x^{-2+k}+\cdots+b_{j} x^{-j+k}
\end{aligned}
$$

for $x^{k} \in R\left[x^{s}\right]$.

Similarly we can define $M\left[\left[x^{s}, x^{-s}\right]\right]$ as an $R\left[x^{s}\right]$-module.

Theorem 3.5. For any nonzero left $R$-module $E, E\left[x^{s}, x^{-s}\right]$ is not an injective left $R\left[x^{s}\right]$-module.

Proof. Define $f:\left(1+x^{2}\right) \longrightarrow E\left[x^{s}, x^{-s}\right]$ by $f\left(1+x^{2}\right)=e$ for $e \in E$ and consider the following diagram

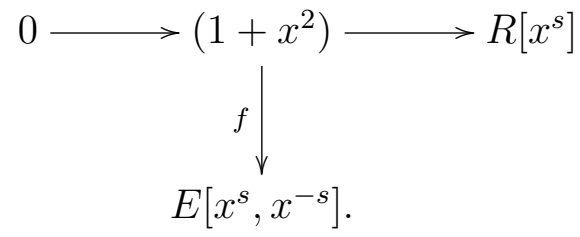

Then we easily see that the above diagram can not be completed for any $R\left[x^{s}\right]$-linear map. Thus $E\left[x^{s}, x^{-s}\right]$ is not an injective left $R\left[x^{s}\right]$ module.

\section{References}

[1] Z. Liu. Injectivity of Modules of Generalized Inverse Polynomials, Comm. Algebra, 29 (2) (2001), 583-592.

[2] A.S. McKerrow. On the Injective Dimension of Modules of Power Series, Quart J. Math., Oxford, 25 (3) (1974), 359-368.

[3] D.G. Northcott. Injective Envelopes and Inverse Polynomials, London Math. Soc. 3 (2) (1974), 290-296.

[4] S. Park. The Macaulay-Northcott Functor, Arch. Math. 63 (1994), 225-230.

[5] S. Park, Gorenstein Rings and Inverse Polynomials, Comm. Algebra, 28 (2) (2000), 785-789.

[6] S. Park, The General Structure of Inverse Polynomial Modules, Czech. Math. J. 51 (126) (2001), 343-349.

[7] S. Park and E. Cho. Injective and Projective Properties of $R[x]$-modules, Czech. Math. J. 54 (129) (2004), 573-578.

[8] S. Park, J. Jeong. Inverse polynomial modules induced by an R-linear map, Bull. Korean Math. Soc. 47 (2010), 693-669.

[9] J. Rotman, An Introduction to Homological Algebra, Academic Press Inc., New York (1979). 
Department of Mathematics

Dong-A University

Pusan 604-714, Korea

E-mail: choeh@donga.ac.kr

Department of Mathematics

Dong-A University

Pusan 604-714, Korea

E-mail: jsjeong@donga.ac.kr 